\title{
Vulvar Superficial Angiomyxoma
}

National Cancer Institute

\section{Source}

National Cancer Institute. Vulvar Superficial Angiomyxoma. NCI Thesaurus. Code C40324.

A vulvar angiomyxoma arising from the dermis or subcutaneous tissues. It may recur following resection. 